\title{
Características socio-demográficas y laborales de los periodistas españoles e índice de satisfacción profesional
}

\author{
Roberto Rodríguez Andrés
}

Profesor Asociado de la Facultad de Comunicación de la Universidad de Navarra

\section{RESUMEN}

La tradición de investigaciones empíricas sobre la naturaleza de la profesión periodística, tan frecuente en otros países, está empezando a consolidarse en España. En el presente artículo, se exponen los principales resultados obtenidos por el Departamento de Comunicación Pública de la Universidad de Navarra tras una encuesta realizada a 1.000 profesionales de la información de toda España, y se comparan con otros estudios realizados en nuestro país. Se describen las características socio-demográficas y laborales de los periodistas y también se analiza el índice de satisfacción que muestran con respecto al trabajo que ejercen, una cuestión que condiciona la forma en la que desarrollan ese trabajo y que influye notablemente en los productos informativos que llegan finalmente al público.

\section{ABSTRACT}

The empirical research tradition on the nature of the journalist profession, so frequent in other countries, is begining to consolidate in Spain. In this article, the main results obtained by the University of Navarra Public Communication Department, by means of a survey carried out with 1.000 information professionals from all over Spain, are presented and compared with other similar studies made in Spain. In the survey the journalist socio demographic and labour characteristics are described and the satisfaction rate in relation with their jobs is shown. This is an important issue that affects the way journalists work and also has an important effect on the final informative products that reach to people.

Palabras claves: Periodismo/Actitudes profesionales de los periodistas/Índice de satisfacción profesional de los periodistas

Key words: Journalism/Journalists professional attitudes/Journalists professional satisfaction rate.

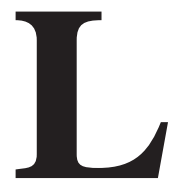

os periodistas dedican su vida profesional a informar. Sin embargo, se muestran reacios cuando se pretende informar sobre ellos, cuando se intenta averiguar cómo son y cómo piensan, cuáles son sus hábitos laborales, sus costumbres y sus rutinas. Cada vez que un equipo de investigación ha pretendido sobrepasar esta coraza profesional se ha encontrado 
con un alto índice de rechazo, aun cuando ese intento haya sido en forma de cuestionario anónimo.

En nuestro país, la tradición de estudios empíricos acerca de la profesión periodística es bastante reciente. En 1976, López Pintor y Santamaría aludían a que por razones de muy diversa índole, y en contra de lo que ocurría en otros países, la sociología de las profesiones y, en especial, de la profesión periodística, apenas se había desarrollado en España ${ }^{1}$. Ortega y Humanes creen que esta escasez de estudios se ha producido por varios factores, entre ellos la falta de tradición en la realización de análisis empíricos sobre la comunicación por parte de los sociólogos y el escaso interés de los periodistas a ser analizados por instancias ajenas a la profesión ${ }^{2}$.

El primer análisis de los informadores españoles basado en sondeos fue el realizado por Demoscopia en 1990 por encargo de la Asociación de la Prensa de Madrid. En total, fueron encuestados más de 700 periodistas de los 5.000 que pertenecían a las entidades encuadradas en la Federación Española de Asociaciones de la Prensa ${ }^{3}$.

A partir de este trabajo, la comunidad científica comenzó a interesarse por este terreno y empezaron a publicarse diferentes investigaciones procedentes de Universidades españolas y también de las propias organizaciones profesionales. Así, en 1991, Martín Algarra y González Gaitano, de la Universidad de Navarra, realizaron un estudio más específicamente centrado en las actitudes profesionales de los periodistas ${ }^{4}$. En 1994, Diezhandino, Bezunartea y Coca, de la Universidad del País Vasco, llevaron a cabo un análisis sociológico de la elite de la profesión (directores y jefes de sección) ${ }^{5}$. En ese mismo año, la Asociación de la Prensa de Madrid volvió a realizar una encuesta sobre los periodistas españoles, que llevó por título El perfil del profesional ${ }^{6}$. La Universidad Complutense de Madrid llevó a cabo en 1995 un cuestionario realizado en entrevista personal sobre "Periodismo y sociedad en la España de hoy", dirigido por Félix Ortega ${ }^{7}$. Apoyado en la misma metodología, en 1999, el CIS difundió el estudio Los profesionales de los medios

1 Cfr. LÓPEZ PINTOR, R. y SANTAMARÍA OSORIO, J. (1976): "Imagen de la profesión periodística en España: comentarios a un sondeo de opinión”, Comunicación XXI, n. 26, pp. 31-37.

2 Cfr. ORTEGA, Félix y HUMANES, Ma Luisa (2000): Algo más que periodistas. Sociología de una profesión, Barcelona: Ariel, pp. 223-224.

3 Cfr. "El periodista español. Retrato intermitente", Periodistas, n. 39, 1990 (diciembre), Servicio de Publicaciones de la Asociación de la Prensa de Madrid.

4 Cfr. MARTÍN ALGARRA, Manuel y GONZÁLEZ GAITANO, Norberto (1994): "La formación de los periodistas españoles en la encuesta Media and Democracy", Estudios de Periodística, n. 3, pp. 49-75.

5 Cfr. DIEZHANDINO, M., BEZUNARTEA, O. y COCA, C. (1994): La elite de los periodistas, Bilbao: Universidad del País Vasco.

6 Cfr. Asociación de la Prensa de Madrid (1994): El perfil del profesional.

7 En este estudio, financiado por la DGICYT, se encuestó a 401 periodistas de prensa, radio, televisión y agencias de la Comunidad de Madrid entre los meses de abril y mayo de 1995. 
de comunicación, elaborado por García de Cortázar, García de León, Ortega, Callejo y Del Val, de las Universidades Complutense y UNED ${ }^{8}$. En el año 2000, se editaron dos trabajos más: el de Ortega y Humanes ${ }^{9}$; y el de García de Cortázar y García de León ${ }^{10}$. A lo largo de todo este tiempo, diversas Asociaciones de la Prensa y Colegios Profesionales han publicado también estudios acerca de las características profesionales de los informadores de sus respectivas regiones ${ }^{11}$. Y estos análisis de carácter más general se vienen a sumar a otra serie de artículos y publicaciones que han analizado también la profesión periodística en nuestro país $^{12}$.

A la vista de estos trabajos se puede afirmar que, a fecha de hoy, la investigación empírica acerca de la profesión periodística cuenta ya con un sólido corpus científico que ha permitido dar a conocer de forma detallada las características generales de los periodistas españoles.

8 Cfr. Los profesionales de los medios de comunicación, estudio n. 2.306 del Centro de Investigaciones Sociológicas (CIS), diciembre 1998-febrero 1999.

9 Cfr. ORTEGA, Félix y HUMANES, Ma Luisa (2000): Algo más que periodistas. Sociología de una profesión, Barcelona: Ariel.

10 Cfr. GARCÍA DE CORTÁZAR, Marisa y GARCÍA DE LEÓN, Ma Antonia (coords.) (2000): Profesionales del Periodismo, Madrid: CIS.

11 Cfr. Entre estos estudios llevados a cabo por organizaciones de periodistas destacan: Colegio de Periodistas de Cataluña (1992, 1996, 2000); Asoc. de la Prensa de Soria (1995); Asoc. de la Prensa de Bizkaia (1999); Asociación de la Prensa de Aragón (2001); Asoc. de la Prensa de Granada (2001); Asoc. de la Prensa de Málaga (2002); Colegio de Periodistas de Galicia (2002). Basados en estos sondeos se han realizado también distintas publicaciones: VV.AA. (1988): "Los periodistas catalanes analizan su situación actual", Periodistas, n. 11; TÚÑEZ, Miguel (1996): "Honestos, obxectivos e independientes", informe sobre la profesión periodística en Galicia, Xornalistas. Revista de la Asociación da Prensa de Santiago de Compostela, n. 1, pp. 16 y sg.

12 Cfr. MARTÍN ALGARRA, Manuel (1992): "La objetividad en los periodistas españoles", Nuestro Tiempo, octubre, pp. 116-125; ORTEGA, Félix (1996): "El ascenso de una nueva clase. Los periodistas en la sociedad española", Telos, n. 47, pp. 21-22; HUMANES, Ma Luisa (1997): La formación de los periodistas en España (tesis doctoral), Madrid: Universidad Complutense de Madrid; HUMANES, Ma Luisa (1998): "La profesión periodística en España", Zer, n. 4, pp. 265-278; HUMANES, Ma Luisa (1998): "La investigación sobre el emisor de la comunicación", Comunicación y Cultura, n. 4, pp. 49-53; CANEL, Ma José y PIQUÉ, Antoni María (1998): "Journalists in Emerging Democracies: The Case of Spain", en WEAVER, D. (ed.): The Global Journalist, New Jersey: Hampton Press; CANEL, Ma José y PIQUÉ, Antoni María (1998): "La evaluación profesional de los periodistas españoles", Comunicación y Sociedad. Revista Mexicana de Comunicación, n. 32, enero-abril, pp. 107-134; CANEL, Ma José y SÁDABA, Teresa (1999): "La investigación académica sobre las actitudes profesionales de los periodistas. Una descripción del estado de la cuestión", Comunicación y Sociedad, vol. XII, n. 2, pp. 9-32; CANEL, Ma José, RODRÍGUEZ ANDRÉS, Roberto y SÁNCHEZ ARANDA, José Javier (1999): "La influencia de las actitudes profesionales de los periodistas españoles en las noticias", Análisi, n. 23, pp. 89-108; SÁNCHEZ ARANDA, José Javier y RODRÍGUEZ ANDRÉS, Roberto (1999): "Profesionalidad y ética. El caso de los periodistas españoles”, Comunicación y Sociedad, vol. XII, n. 2, pp. 93-114; GARCÍA TÓJAR, Luis (1999): El factor humano. Socioanálisis de los productores de la información periodística en España (tesis doctoral), Madrid: Universidad Complutense de Madrid. 


\section{1.- Objetivos y metodología}

Para continuar la estela de análisis anteriores y para seguir profundizando en el conocimiento de la profesión periodística de nuestro país, el departamento de Comunicación Pública de la Universidad de Navarra, en colaboración con el profesor David Weaver, de la Universidad de Indiana, trabajó entre 1995 y 1997 en la elaboración de un sondeo que permitiera obtener una radiografía aproximada de la realidad periodística española. Ese sondeo fue remitido a una muestra aleatoria compuesta por 1.000 periodistas, que se dividieron proporcionalmente según el tipo de medio (prensa, radio o televisión); según el ámbito en el que desempeñaban su trabajo (nacional, regional o local); y según la propiedad del medio (pública o privada) ${ }^{13}$.

En ese sondeo, completamente anónimo, se incluyeron 53 preguntas. El índice de respuesta obtenido fue del 29,3 por ciento, un dato que se sitúa en la media de los estudios de este tipo ${ }^{14}$. Esta investigación cuantitativa se completó con otra de carácter cualitativo, a través de entrevistas en profundidad a varios directivos de medios de comunicación que, por su experiencia, tuvieran buen conocimiento del trabajo en las redacciones ${ }^{15}$. Los principales resultados de esta investigación fueron publicados por el Centro de Investigaciones Sociológicas en diciembre de $2000^{16}$.

El estudio se diseñó para conocer las características personales y profesionales de los periodistas españoles. Sin embargo, pretendía ir más allá. Al iniciar la investigación, se partía de la ya extendida creencia de que los medios de

13 La muestra seleccionada suponía un nivel de confianza del 95,5 por ciento, y $\mathrm{P}=\mathrm{Q}$, y un margen de error de más menos 3 por ciento. Los 1.000 periodistas se dividieron proporcionalmente según prensa, radio o televisión. Según datos de la Asociación de la Prensa, la proporción entre estos medios es de 60 por ciento prensa, 20 por ciento televisión y 20 por ciento radio. Así se procedió con la muestra, con lo que se enviaron 600 cuestionarios a periodistas de prensa, 200 a televisión y 200 a radio. La estratificación por medios, siguiendo los datos del EGM y de la OJD, fue la siguiente. En prensa: 50 por ciento a diarios regionales y otro 50 por ciento a diarios de Madrid y Barcelona (30 y 20 respectivamente). Para la radio: 60 por ciento de emisoras privadas y 40 de públicas. En televisión: 73 por ciento de cadenas públicas y 27 por ciento de privadas, con un 41,5 por ciento de autonómicos y un 58,5 por ciento de acción nacional.

14 Como ya se ha mencionado, la profesión periodística suele ser muy reacia a contestar a este tipo de sondeos, lo que se traduce en escasos índices de respuesta por parte de los encuestados. Este problema ha sido común a todos los estudios realizados hasta el momento. De hecho, ya en 1990, en el primer estudio empírico realizado sobre la profesión periodística española, los analistas de Demoscopia reconocían en su informe que habían encontrado "un nivel de rechazo muy superior al que en otros colectivos socioprofesionales solemos encontrar" (Cfr. "El periodista español. Retrato intermitente", Periodistas, n. 39, 1990 (diciembre), Servicio de Publicaciones de la Asociación de la Prensa de Madrid).

15 Las entrevistas fueron semiestructuradas: en todas se utilizó un guión que tenía por objetivo recibir el feedback de los directivos a los resultados arrojados por el sondeo. Todas las entrevistas, con una duración media de dos horas y media, fueron grabadas y transcritas. En este artículo se hará referencia a declaraciones de Juan Luis Cebrián (El País), Francisco Jiménez Alemán (entonces, director de $A B C$ ), Fernando Ónega (en el momento de la entrevista en Antena 3), José Antonio Zarzalejos (cuando se encontraba en el Grupo Correo) y Luis Fernández (en el momento de la entrevista, en Tele 5).

16 Cfr. CANEL, Ma José, RODRÍGUEZ ANDRÉS, Roberto y SÁNCHEZ ARANDA, José Javier (2000): Periodistas al descubierto, Madrid: CIS. 
comunicación no son meros espejos, meros transmisores de la realidad, sino mediadores e intérpretes de la misma ${ }^{17}$. Por tanto, el hecho de conocer cuáles son los rasgos culturales y sociales más destacables de los periodistas cobra aún mayor importancia. Si se conocen esas características, se podrán explicar de forma más eficaz los motivos que conducen a los informadores a desarrollar un determinado tipo de mediación o interpretación de la realidad.

Profundizar en las características socio-demográficas de los periodistas (edad, sexo, nivel de educación, formación continuada) o en sus condiciones laborales (sueldo y horarios) permitirá conocer algo más de cómo desarrollan esa tarea de mediación. Ese será, pues, el objetivo del presente artículo. Además, nos detendremos de forma especial en uno de los elementos que, bajo nuestro punto de vista, más influye en las actitudes profesionales de los informadores y, por ende, en su labor mediadora: el índice de satisfacción con respecto al trabajo que ejercen.

A lo largo del artículo se irán exponiendo los resultados del análisis llevado a cabo en el seno del departamento de Comunicación Pública de la Universidad de Navarra, incidiendo igualmente en la evolución que ha ido viviendo la profesión a la vista de los resultados que se han obtenido en otros estudios de estas características $^{18}$.

\section{2.- Características socio-demográficas de los periodistas españoles}

\section{1.- La edad de los periodistas}

Los periodistas españoles son preferentemente jóvenes. Según nuestro sondeo, la media de edad de nuestros informadores es de 34 años, muy similar a la observada en otros países. Los periodistas estadounidenses rondan los 36. Los británicos y alemanes, los 38. Los franceses, canadienses y finlandeses, los 40 . Caso especial es el de los periodistas australianos, que apenas llegan a los 32 años de media de edad ${ }^{19}$.

Comparando estos datos con los sondeos precedentes, advertimos un constante rejuvenecimiento de la profesión en nuestro país. En 1990, los periodistas tenían alrededor de 41 años $^{20}$. En el 94, la media bajó hasta los $40^{21}$. Hoy, según nuestro estudio, es de 34 .

Por tramos de edad, se aprecia que uno de cada dos periodistas tiene entre 25 y 35 años, es decir, que la mitad de los profesionales está todavía en la edad

17 Cfr. SHOEMAKER, Pamela y REES, Stephen (1996): Mediating the Message, Nueva York: Longman.

18 Al estar todos estos estudios apoyados en muestras diferentes, la comparación no permite conclusiones estadísticamente válidas. Sin embargo, consideramos de interés presentar estas comparaciones, pues permiten conocer cuáles son las tendencias de la evolución de la profesión periodística.

19 Cfr. WEAVER, D. y WILHOIT, C. (1996): The American Journalist, New Jersey: Lawrence Erlbaum; WEAVER, D. (ed.) (1998): The Global Journalist, New Jersey: Hampton Press.

20 Cfr. "El periodista español. Retrato intermitente", Periodistas, n. 39, 1990 (diciembre), Servicio de Publicaciones de la Asociación de la Prensa de Madrid.

21 Cfr. Asociación de la Prensa de Madrid (1994): El perfil del profesional. 
en la que se están aprendiendo y desarrollando las habilidades básicas. Tan sólo un 11,5 por ciento tiene más de 45 años, edad considerada en otras profesiones como la más fértil y productiva a nivel intelectual.

¿Cuáles son los motivos de este constante rejuvenecimiento? Se podrían alegar aquí razones históricas. En España, el desarrollo de los medios de comunicación y de la profesión periodística ha sido reciente. Hasta la llegada de la democracia no empezaron a proliferar nuevos periódicos, revistas y emisoras de radio. El régimen de Franco había controlado férreamente tanto el número y propiedad de estos medios como la propia incorporación a la profesión ${ }^{22}$. Con este panorama, el número de interesados por el periodismo era reducido. Además, no fue hasta los años 50 y 60 cuando el periodismo se incorporó a las Universidades españolas en forma de facultades, con lo que fue a partir de entonces cuando comenzaron a abrirse nuevas vías para que los jóvenes se adentraran en el mundo del periodismo ${ }^{23}$.

Sin embargo, la democracia y, con ella, la eclosión de los medios y la posibilidad de escribir y contar en un ambiente de libertad, dio un nuevo impulso a esta profesión. Empezaron a surgir nuevas Facultades de Ciencias de la Información y, poco a poco, gran cantidad de jóvenes fue incorporándose al periodismo.

Esta situación de constante rejuvenecimiento está siendo observada desde los propios medios con cierta preocupación, según manifestaron los directivos entrevistados, ya que está cambiando los hábitos y formas de trabajo de los medios, y también su función mediadora. Como afirmaron Cebrián y Onega, cualquier oficio necesita de la madurez y sabiduría que imparten los veteranos, puesto que son ellos quienes pueden imponer la calma precisa para contrarrestar el entusiasmo de la juventud. En la medida en que la profesión vaya perdiendo a sus veteranos irá perdiendo igualmente ese punto de apoyo.

\section{2.- Incremento del número de mujeres}

En España, tan sólo un 34 por ciento del total de los periodistas son mujeres. La relativa escasa presencia de mujeres no es exclusiva de nuestro país. En Estados Unidos, Australia, China o Hungría, el porcentaje es el mismo. Y hay otros lugares en los que la participación femenina es aún mucho menor, como Francia con un 20 por ciento, Gran Bretaña 25, Alemania 30, Canadá 28 o México $25^{24}$.

22 Cfr. SÁIZ, Ma Dolores y SEOANE, Ma Cruz (1983): Historia del Periodismo en España, Madrid: Alianza; SÁNCHEZ ARANDA, José Javier y BARRERA, Carlos (1992): Historia del periodismo español, Pamplona: Eunsa; BARRERA, Carlos (1995): Periodismo y franquismo, Barcelona: Ediciones Internacionales Universitarias; FUENTES, J.F. y FERNÁNDEZ, J. (1997): Historia del periodismo español, Madrid: Síntesis.

23 Respecto a la aparición de los estudios de periodismo y específicamente su introducción en la Universidad, cfr. BARRERA, Carlos (ed.) (1999): Del gacetero al profesional del periodismo, Madrid: Fragua.

24 Cfr. WEAVER, D. y WILHOIT, C. (1996): The American Journalist, New Jersey: Lawrence Erlbaum; WEAVER, D. (ed.) (1998): The Global Journalist, New Jersey: Hampton Press. 
A pesar de que este porcentaje es todavía exiguo, hay que subrayar que la incorporación de la mujer está siendo progresiva. Si se observan sondeos anteriores, en 1990 el porcentaje de mujeres periodistas apenas llegaba al 17 por ciento ${ }^{25}$. En 1994, había alcanzado el $25^{26}$. Y hoy, como acabamos de anotar, supone ya un 34 por ciento.

Esta mayor presencia de la mujer en el periodismo se observa también en las responsabilidades que van adquiriendo en la redacción. Hoy por hoy, la mujer está trabajando en todas las secciones, y desempeñando exactamente los mismos cometidos que los hombres (hay mujeres redactoras, columnistas, presentadoras, comentaristas deportivas, cronistas políticas, reporteras de guerra...). Sin embargo, hay aún dos aspectos en los que siguen existiendo desigualdades en nuestro país. En primer lugar, el número de mujeres directoras o redactoras jefe es aún reducido. Su incorporación generalizada a los puestos directivos todavía no es una realidad $^{27}$. Y en segundo lugar, las mujeres siguen cobrando menos que los hombres, a pesar de que se encuentren en los mismos niveles y categorías laborales y aun cuando lleven ejerciendo el mismo número de años de experiencia profesional, aunque en este aspecto no hay coincidencia entre todos los estudios demoscópicos realizados hasta el momento en nuestro país ${ }^{28}$.

El aumento del número de mujeres en la profesión se está produciendo sobre todo en los tramos correspondientes a jóvenes, debido a su incorporación masiva a las Facultades de Ciencias de la Información. Hoy por hoy, entre los periodistas jóvenes hay más mujeres que hombres (por ejemplo, en el grupo de edad 21-25 años, hay un 58 por ciento de mujeres y un 42 por ciento de hombres.

25 Cfr. "El periodista español. Retrato intermitente", Periodistas, n. 39, 1990 (diciembre), Servicio de Publicaciones de la Asociación de la Prensa de Madrid.

26 Cfr. Asociación de la Prensa de Madrid (1994): El perfil del profesional.

27 Esta situación se ha puesto en evidencia también en otros estudios. En la encuesta de 1990, había un 27\% de hombres directivos, un $20 \%$ de redactores-jefe y un $8 \%$ de jefes de sección; unos datos que bajaban hasta el 12, 15 y 5\%, respectivamente, en el caso de las mujeres (Cfr. "El periodista español. Retrato intermitente", Periodistas, n. 39, 1990 (diciembre), Servicio de Publicaciones de la Asociación de la Prensa de Madrid). Y en el informe del CIS de 1999, un 81,5\% de los encuestados creía que había pocas o muy pocas mujeres ocupando cargos o puestos de responsabilidad en la profesión periodística, lo que deja entrever que entre los propios periodistas existe una clara convicción de que las mujeres aún no han conseguido equipararse a los hombres en el desempeño de funciones directivas. Además, un $66,7 \%$ de 10 s encuestados creía que las mujeres tenían menos apoyo que los hombres por parte de las empresas para ocupar estos cargos y un 37,5\% reconocía abiertamente que en esta profesión no existe igualdad de oportunidades (Cfr. Los profesionales de los medios de comunicación, estudio n. 2.306 del Centro de Investigaciones Sociológicas (CIS), diciembre 1998-febrero 1999, preguntas 26, 26b y 27, pp. 17-18).

28 En el sondeo del Departamento de Comunicación pública de la Universidad de Navarra, de 1997, no se apreciaban diferencias significativas con respecto a los sueldos de hombres y mujeres. Ambos sexos cobraban cantidades similares (Cfr. CANEL, Ma José, RODRÍGUEZ ANDRÉS, Roberto y SÁNCHEZ ARANDA, José Javier (2000): Periodistas al descubierto, Madrid: CIS). Sin embargo, en la encuesta del CIS de 1999, se apreciaba que dentro de las mismas edades, las mujeres siempre perciben menos ingresos que los varones (Cfr. GARCÍA DE CORTÁZAR, Marisa y GARCÍA DE LEÓN, Ma Antonia (coords.) (2000): Profesionales del Periodismo, Madrid: CIS, p. 53). 


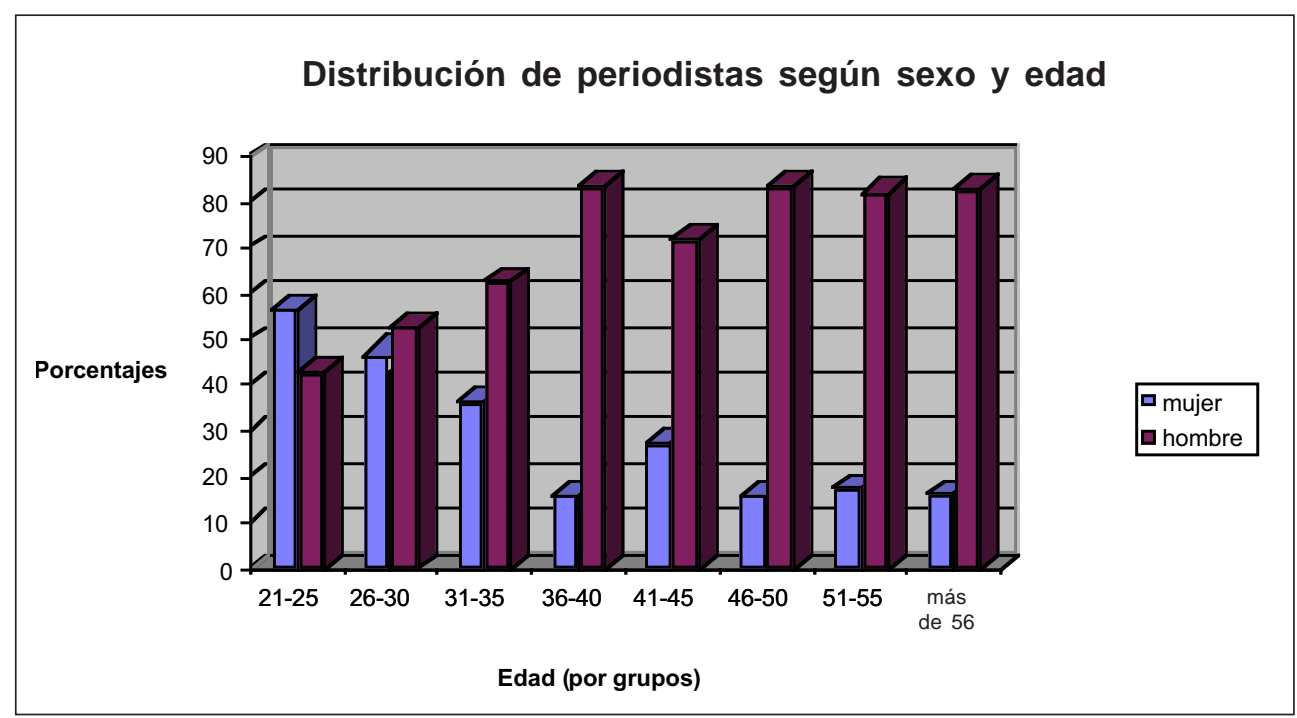

Y de forma global, es destacable que el 78 por ciento de las mujeres periodistas sean menores de 35 años).

Con estos datos, podría pensarse que en unos cuantos años la tendencia se habrá invertido, y la profesión periodística habrá pasado a ser mayoritariamente femenina. Sin embargo, la realidad profesional nos muestra que un buen número de mujeres se retira de este trabajo antes de los 40 años, para dedicarse a otros cometidos más compatibles con la familia ${ }^{29}$, con lo que el predominio masculino, aún siendo cada vez menor, podría continuar en el futuro. A lo largo de su carrera profesional, periodistas como Ónega, Cebrián o Zarzalejos han ido observando este fenómeno. Como afirmaron en entrevistas mantenidas a partir de los datos de nuestro sondeo, el ritmo de vida del periodismo hace que muchas mujeres acaben decidiendo no hacerla compatible con la familia. Sin embargo, estos propios periodistas reconocen que en los últimos años esta situación está cambiando. Circunstancias sociológicas como el reparto de tareas domésticas entre hombres y mujeres han propiciado que la mujer pueda continuar normalmente su actividad profesional aun después de tener hijos.

29 Así lo constataba, por ejemplo, la encuesta del Centro de Investigaciones Sociológicas de 1999. En este estudio, los propios encuestados manifestaban que las mujeres periodistas cuentan con un mayor número de responsabilidades familiares que los hombres y con menor disponibilidad horaria (Cfr. Los profesionales de los medios de comunicación, estudio n. 2.306 del Centro de Investigaciones Sociológicas (CIS), diciembre 1998-febrero 1999, pregunta 29, p. 19). 


\section{3.- Nivel de estudios y formación continuada}

Pulitzer, maestro de periodistas, luchó durante buena parte de su vida para institucionalizar los estudios de periodismo y para crear escuelas especializadas que enseñaran esta práctica profesional ${ }^{30}$. Sus esfuerzos no fueron en balde ya que, a lo largo del siglo XX, el periodismo se fue incorporando como una disciplina más dentro de las Universidades de todo el mundo, con lo que se ha ido consolidando un corpus científico que apoya a las prácticas profesionales. Esto ha permitido que la formación de los periodistas se haya incrementado con el paso de los años.

¿Cuál es la formación de los periodistas españoles? Según nuestro sondeo, tan sólo un 1,7 por ciento de los profesionales tiene únicamente estudios elementales o de bachiller. O lo que es lo mismo: el 98,3 por ciento restante tiene alguna preparación universitaria (algunos cursos, título superior, master o doctorado). En líneas generales, por tanto, los periodistas españoles en comparación con los de otros países se caracterizan por un alto nivel de estudios, circunstancia que sin duda alguna influye, y positivamente, en la forma en que llevan a cabo esa mediación e interpretación de la realidad a la que aludíamos al inicio del artículo. Un periodista mejor formado tendrá mayor capacidad de decisión, de valoración y de análisis, y podrá ofrecer un mejor servicio a la sociedad.

En cuanto a la formación específica en el ámbito de la comunicación, es destacable que un 92,1 por ciento de los que poseen título universitario sí tiene este tipo de estudios (bien a través de Escuelas de Periodismo o a través de licenciaturas o masters ${ }^{31}$. Este dato es sumamente importante porque, de hecho, convierte a los periodistas españoles en los que mejor formación periodística tienen de todo el mundo, muy por encima de los porcentajes de otros países como Brasil con un 84 por ciento, Estados Unidos 39, China y Francia 32 o Gran Bretaña $4^{32}$.

Este aspecto guarda estrecha relación con la polémica acerca del intrusismo laboral en el mundo del periodismo. Hace pocos años, los estudiantes de Ciencias de la Información de varias facultades españolas salieron a la calle para protestar

30 Cfr. NAVAS, Alejandro y SÁNCHEZ, José Francisco (1995): "La formación de los profesionales de la comunicación”, Situación, n. 4, pp. 279-294, p. 279; SÁNCHEZ ARANDA, José Javier (1998): Pulitzer. Luces y sombras en la vida de un periodista genial, Pamplona: Eunsa, pp. 226 y sg.

31 Este dato ha crecido también con el paso de los años. En 1990, en la encuesta de la Asociación de la Prensa de Madrid, el $66 \%$ de los periodistas tenía formación específica en Comunicación, bien a través de las Escuelas de Periodismo o de las Licenciaturas en Ciencias de la Información (Cfr. "El periodista español. Retrato intermitente", Periodistas, n. 39, 1990 (diciembre), Servicio de Publicaciones de la Asociación de la Prensa de Madrid). Esta cifra aumentó en la encuesta de 1995 de la Universidad Complutense de Madrid, donde el $85 \%$ de los entrevistados era licenciado en Ciencias de la Información (Cfr. HUMANES, M ${ }^{a}$ Luisa (1998): “La profesión periodística en España”, Zer, n. 4, pp. 265-278, p. 268).

32 Cfr. WEAVER, D. y WILHOIT, C. (1996): The American Journalist, New Jersey: Lawrence Erlbaum; WEAVER, D. (ed.) (1998): The Global Journalist, New Jersey: Hampton Press. 
por la creciente presencia de profesionales de otras carreras en los medios de comunicación. Reclamaban que para trabajar en un periódico, en una radio o en una televisión, se exigiera estar en posesión del título de periodista. Vistos los resultados del sondeo, parece que ese intrusismo está empezando a desaparecer, puesto que los medios confían mayoritariamente en los licenciados en Comunicación $^{33}$ y la abundancia de demandantes facilita que cumplan esa condición.

Tras estos datos, cabría concluir este apartado subrayando el buen nivel de formación que atesoran los periodistas españoles. Sin embargo, el problema es que este buen nivel inicial no suele ser acrecentado con el paso del tiempo. En contraste con otros profesionales, que perfeccionan continuamente sus saberes, es destacable que uno de cada dos periodistas no ha recibido jamás formación de reciclaje, lo cual significa que la mitad de los informadores no ha vuelto a frecuentar un aula desde que abandonó la Universidad. En cuanto a lectura de revistas especializadas en temas de comunicación, tan sólo un 34,6 por ciento de la muestra consulta de forma habitual este tipo de publicaciones.

¿A qué se debe esta escasa predisposición al estudio o a la formación continuada? Principalmente al tiempo. Los periodistas afirman estar demasiado ocupados para poder realizar esta actualización de saberes. Pero si pudieran, desearían mejorar su formación en los siguientes campos: idiomas 52,1 por ciento, periodismo de investigación 16,9 , informática 14,6 , diseño 6,9 y recursos humanos 6,5 .

\section{3.- Las condiciones laborales de los periodistas y el índice de satisfacción profesional}

Tras analizar las características más generales de los periodistas españoles, nos adentramos a continuación en un aspecto mucho más subjetivo: conocer su grado de satisfacción o insatisfacción con respecto a la profesión que ejercen. Esta cuestión suscita a menudo el debate entre los propios periodistas. Muchos son los que se quejan pero, a la vez, muy pocos son los que abandonan el periodismo activo. Los informadores protestan por los sueldos tan bajos que perciben y por los intempestivos horarios que les exige su trabajo. Pero, a la vez, vibran cada vez que obtienen una exclusiva, y no dudan en perder días enteros detrás de una buena información. Entonces, ¿están realmente contentos los periodistas con su profesión? Y si no es así, ¿cuáles son los motivos que justifican esa insatisfacción?

33 Los directores de medios de comunicación entrevistados tras la elaboración de nuestro sondeo estuvieron de acuerdo a la hora de subrayar que los formados en Comunicación son los mejor cualificados para contar las historias a los ciudadanos. Sin embargo, todos ellos reconocieron también que, a veces, en determinados temas, se hace necesaria la participación y colaboración de expertos para no cometer errores. De esta forma, los medios contratan también a médicos, abogados o economistas, a los que se les entrena en las destrezas redaccionales, para este tipo de informaciones más comprometidas o especializadas. 
Con el fin de medir la satisfacción laboral se siguió el criterio empleado por Barbasch $^{34}$ y por Barrett ${ }^{35}$ : evitar la pregunta directa. La experiencia adquirida en sondeos anteriores ha servido para poner de relieve que difícilmente se obtienen resultados objetivos de insatisfacción si se formula la pregunta "¿Está usted satisfecho con su profesión?". Es demasiado rotunda y directa como para ser contestada abiertamente. Además, reconocer esa insatisfacción puede denotar por parte del que contesta cierto índice de frustración o fracaso. Por este motivo se usó una pregunta indirecta: “¿Ha pensado dejar la profesión?”, con una escala de tres valores: "nunca", "alguna vez" y "muchas veces".

Las respuestas fueron las siguientes: nunca 31,2 por ciento; alguna vez 51,1 por ciento; muchas veces 17,7 por ciento. Estos datos muestran un alto índice de insatisfacción entre los periodistas españoles. En concreto, tan sólo el 31,2 por ciento, es decir, tan sólo tres de cada diez, manifestaba no haber pensado nunca en la posibilidad de abandonar esta profesión. El restante 68,8 , es decir, los otros siete de cada diez, reconocieron haberlo pensado alguna vez o, incluso, en muchas ocasiones.

Esta cifra de insatisfacción ha crecido con el paso de los años. En la encuesta de la Asociación de la Prensa de Madrid de 1990, aún no se apreciaba descontento profesional por parte de los periodistas. En el informe de esta encuesta se decía que existía entonces "un nivel de satisfacción razonable con las condiciones de ejercicio del desempeño profesional en el propio medio, con excepciones marcadas en cuanto a la crítica respecto al nivel salarial y la composición de la plantilla" ${ }^{36}$. Sin embargo, cuatro años más tarde, en la encuesta llevada a cabo en 1994 por Diezhandino, Bezunartea y Coca, el tanto por ciento de periodistas insatisfechos subía ya al 53 por ciento ${ }^{37}$. Y estudios posteriores al nuestro han insistido también en el alto nivel de insatisfacción profesional. En 1999, García Tójar publicó, tras realizar 60 entrevistas en profundidad a periodistas, que estos "manifiestan importantes niveles de frustración o descontento en relación con su trabajo" 38 . Y en la encuesta del CIS de 1999, se afirmaba que el 39,8\% de los

34 Cfr. BARBASCH, Jack (1976): "Job satisfaction attitudes surveys", Organization for Economic Cooperatioin and Development, París, pp. 22-23.

35 Cfr. BARRETT, Grace (1984): “Job satisfaction among newspaper women”, Journalism Quarterly, n. 61, pp. 593-599.

36 "El periodista español. Retrato intermitente", Periodistas, n. 39, 1990 (diciembre), Servicio de Publicaciones de la Asociación de la Prensa de Madrid.

37 Cfr. DIEZHANDINO, M., BEZUNARTEA, O. y COCA, C. (1994): La elite de los periodistas, Bilbao: Universidad del País Vasco, pp. 160-166. Esta cifra debe ser observada con reservas puesto que este estudio de 1994 fue realizado entre la elite de los periodistas (directores y jefes de sección), que probablemente estén más satisfechos que el periodista medio pues han llegado más lejos en la profesión y su estabilidad laboral está más garantizada.

38 GARCÍA TÓJAR, Luis (1999): El factor humano. Socioanálisis de los productores de la información periodística en España (tesis doctoral), Madrid: Universidad Complutense de Madrid. 
periodistas españoles había pensado alguna vez en dejar de trabajar en los medios de comunicación ${ }^{39}$.

Por tanto, se observa que los periodistas españoles no están enteramente contentos con su profesión, algo que sin duda influye en el desarrollo de su trabajo y en la forma de tratar e interpretar la realidad. Esta insatisfacción contrasta con los datos de otros países. En Alemania, por ejemplo, un 95 por ciento dice estar satisfecho con su trabajo. En Francia el 87 por ciento, en Canadá el 85, en el Reino Unido el 82 y en Estados Unidos el $77^{40}$.

¿A qué se debe esta situación? A los periodistas encuestados se les planteó la posibilidad de que anotaran las razones por las que habían pensado dejar la profesión. Las respuestas fueron las siguientes:

\section{En caso de que haya pensado dejar la profesión, Porcentajes ¿cuáles son las razones?}

Para estar más con la familia y amigos

Para disminuir el estrés

Para mejorar la situación económica

Por frustración

Para dedicarme a la docencia o a escribir libros $\quad 21,7$

Para hacer otra cosa más interesante 21,1

Por aburrimiento $\quad 6,3$

Por exceso de competencia $\quad 5,2$

Las razones más aducidas tienen que ver con la necesidad de disponer de más tiempo libre. Los periodistas desearían dedicar más horas a sus familiares y amigos. Desearían tener horarios más flexibles que les permitieran combatir el estrés. Les gustaría desempeñar trabajos más relajados, como la docencia, o mejor remunerados económicamente. Sin embargo, llama la atención que, a pesar de

39 Cfr. Los profesionales de los medios de comunicación, estudio n. 2.306 del Centro de Investigaciones Sociológicas (CIS), diciembre 1998-febrero 1999, pregunta 33, p. 22. En esta encuesta de 1999, se incluía otra pregunta que dejaba traslucir el índice de insatisfacción de los periodistas. Preguntados por si alentarían a sus hijos a dedicarse al ejercicio profesional del periodismo, un $55,1 \%$ decía que no, un claro síntoma de que no quieren para sus hijos el tipo de trabajo que ellos desarrollan (Cfr. Los profesionales de los medios de comunicación, estudio n. 2.306 del Centro de Investigaciones Sociológicas (CIS), diciembre 1998-febrero 1999, pregunta 47, p. 30.

40 Cfr. WEAVER, D. y WILHOIT, C. (1996): The American Journalist, New Jersey: Lawrence Erlbaum; WEAVER, D. (ed.) (1998): The Global Journalist, New Jersey: Hampton Press. 
todo, los periodistas no se aburren. Tan sólo un 6,3 por ciento manifestó querer dejar la profesión por aburrimiento ${ }^{41}$.

Estos datos aportan ya alguna luz sobre los motivos de insatisfacción de los periodistas españoles. Sin embargo, no conviene perder de vista que, como ocurre en la mayor parte de los estudios demoscópicos, una cosa son las respuestas a preguntas directas (como es el caso de ésta sobre los motivos de insatisfacción) y otra, a veces bien distinta, los resultados reales que se infieren tras cruzar determinadas variables. Por eso, se hace preciso ofrecer nuevos datos que ayuden a comprender de una forma más precisa las razones de insatisfacción de los periodistas y, sobre todo, que permitan comprobar hasta qué punto es cierto que la falta de tiempo o la escasez de sueldos son los motivos fundamentales de esta situación.

\section{1.- Satisfacción y situación económica}

Uno de los elementos que suelen tenerse en cuenta a la hora de valorar la satisfacción profesional es el salario recibido. A este respecto, la clase periodística se debate entre las cuentas multimillonarias de las grandes estrellas y los salarios más modestos de la gran mayoría de los informadores.

Según nuestro sondeo, la mayoría de los periodistas españoles cobran al mes entre 200.000 y 300.000 pesetas de media, un sueldo considerado como normal también en otras profesiones. Sin embargo, el número de informadores con sueldos inferiores a 200.000 pesetas es bastante más abultado que el que lo hace por encima de las 300.000. Es más, hay un buen grupo que percibe cantidades que podríamos considerar como bajas o muy bajas: un 11,9 por ciento de los periodistas cobra entre 80.000 y 120.000 pesetas, y un 6,1 por ciento no llega a las 80.000 pesetas al mes.

Si el sueldo influyera en la satisfacción laboral, los periodistas jóvenes serían sin lugar a dudas los más insatisfechos. Los sueldos que perciben los recién incorporados a la profesión son muy escasos y, además, sus contratos suelen ser precarios. Entre los menores de 25 años, un 31,6 por ciento cobra menos de 80.000 pesetas al mes y un 42,1 entre 80.000 y 120.000. Es decir, que la nómina de siete de cada diez jóvenes menores de 25 años no llega a las 120.000 pesetas a final de mes. En el siguiente tramo de edad, entre 25 y 30 años, la situación no es muy distinta. En este caso, el 70 por ciento percibe cantidades inferiores a las 200.000

41 Estos datos son muy similares a los obtenidos por la encuesta del CIS de 1999. En este estudio, las razones aducidas para abandonar la profesión eran las siguientes: disminuir el estrés $(51,4 \%)$; tener más tiempo para la familia $(42,4 \%)$; hacer algo distinto o un trabajo más interesante $(28,3 \%)$; dedicarse a la docencia o escribir libros (16,9\%); mejorar su situación económica (13,6\%); eliminar la frustración que le produce $(9,7 \%)$; por aburrimiento $(5,2 \%)$; por otras razones (6,8\%) (Cfr. Los profesionales de los medios de comunicación, estudio n. 2.306 del Centro de Investigaciones Sociológicas (CIS), diciembre 1998-febrero 1999, pregunta 34a, p. 22). Tanto en nuestro estudio como en el del CIS las dos respuestas más frecuentes son disminuir el estrés y estar más con la familia, y la razón menos aducida también en ambos trabajos es la del aburrimiento. 


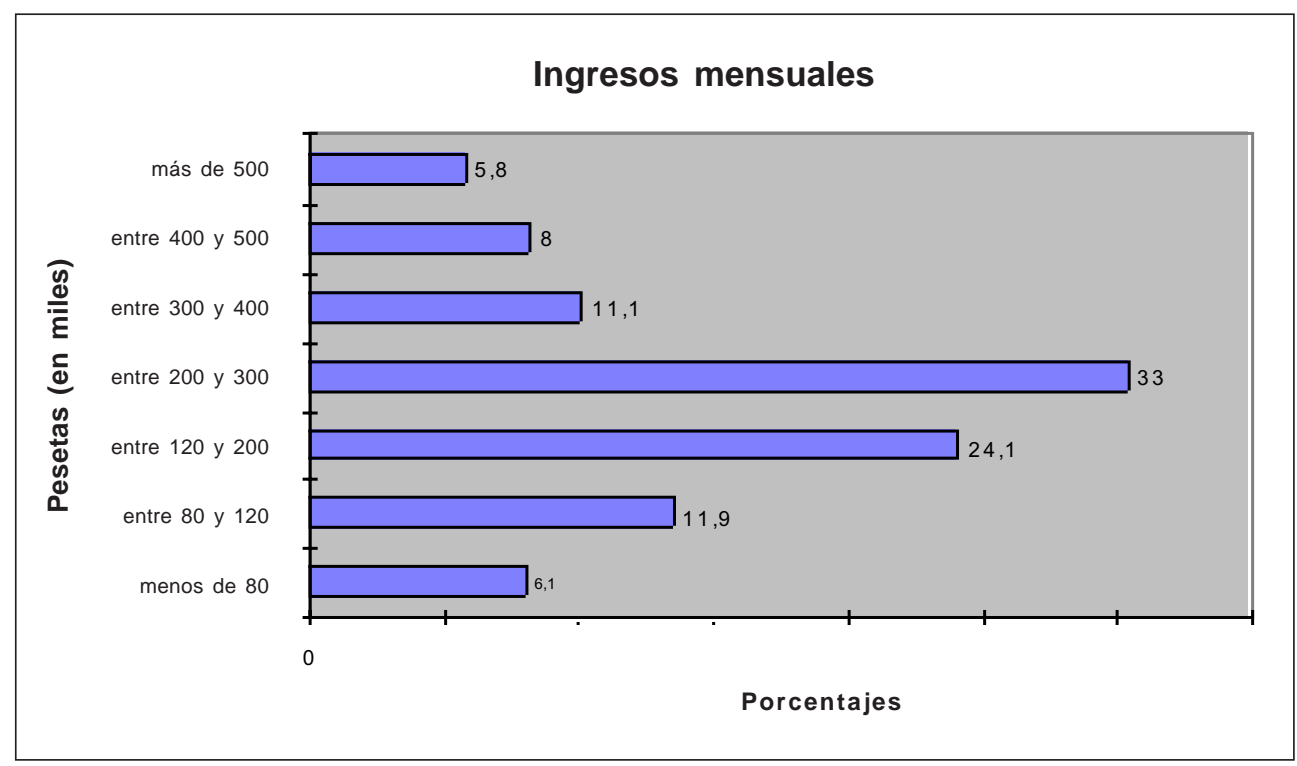

pesetas. Conforme avanzamos en grupos de edad, las condiciones salariales van incrementándose.

¿Es el sueldo un factor decisivo en la insatisfacción profesional de los periodistas españoles? Indudablemente, cobrar poco es siempre motivo de insatisfacción, también para los periodistas. Pero, según los datos de nuestro sondeo, no parece ser el factor más decisivo. El periodismo es una profesión que proporciona un buen número de compensaciones más allá de la mera remuneración económica. Como afirma Santos, el atractivo fundamental de este trabajo no reside en el dinero. La vida del periodista cuenta con la ventaja de ser escasamente rutinaria. Cada día es distinto, con nuevos asuntos y nuevos protagonistas. Además, los informadores están en contacto permanente con la actualidad y tienen el privilegio de ser los primeros en enterarse de las noticias. Pueden tratar de tú a tú a destacadas personalidades de la vida política, cultural, económica o deportiva. Y, por si esto fuera poco, esta profesión ofrece también altas dosis de protagonismo social $^{42}$. Estos factores hacen que el periodista cuente con compensaciones adicionales a la mera retribución salarial. Y quizá por este motivo, el dinero, aun siendo importante, no es el factor decisivo para explicar la insatisfacción de los periodistas españoles.

De hecho, el carácter netamente vocacional de este trabajo y esas compensaciones adicionales hacen que muchos periodistas prefieran seguir en los medios

42 Cfr. SANTOS, Félix (1995): Periodistas, Madrid: Temas de Hoy, p. 90. 
de comunicación con sueldos muy escasos (y otros incluso sin cobrar) antes que buscar otros trabajos alejados del periodismo. Sin embargo, hay que anotar que esta situación está degradando las condiciones laborales de muchos informadores, sobre todo de los más jóvenes, con lo que la exigüidad de sueldos como motivo de insatisfacción, que ahora no es factor decisivo, podría cobrar más protagonismo en los próximos años. "Con la revitalización de esta profesión en los últimos tiempos -afirma Luis Fernández en la entrevista realizada a partir de los datos de nuestro estudio- hemos ido cada vez a peor tipo de contratos. Hay miles de jóvenes en paro dispuestos a trabajar por cuatro duros. Y aún hay muchos más que trabajan incluso sin cobrar, como por ejemplo los estudiantes en prácticas. Los contratos basura en periodismo se han generalizado".

\section{2.- Satisfacción y horarios}

Además del salario, otro de los elementos que suele ser mencionado como factor de insatisfacción entre los periodistas es el de los estirados horarios que impone esta profesión. "Las largas jornadas son algo propio de este trabajo -dice Jiménez Alemán-. Es igual que un cirujano cuando tiene una operación complicada. Se tiene que tirar horas y horas metido en el quirófano, y no se puede marchar hasta que la operación ha concluido. Al periodista le ocurre lo mismo. Hasta que no acabas la noticia no puedes irte a casa. Además, vivimos pendientes de la actualidad. Si a las tres de la mañana se produce una explosión, hay que levantarse para cubrir la noticia".

¿Cuáles son los horarios de los periodistas españoles? Si tenemos en cuenta que las jornadas de trabajo consideradas como normales en nuestro país rondan las 40 horas semanales (es decir, ocho horas diarias), los periodistas superan con creces este baremo. Casi la mitad, un 48,4 por ciento, trabaja normalmente entre ocho y diez horas al día. Y otro 22,7 por ciento supera incluso esa barrera de las diez horas. En resumen, y sumando ambos porcentajes, el 70 por ciento de los periodistas españoles, siete de cada diez, tienen jornadas que superan ampliamente las ocho horas diarias consideradas como normales en otras profesiones.

Hay que tener en cuenta que los hábitos de vida de los españoles influyen de forma decisiva en estos prolongados horarios. Como dice Luis Fernández, "en Alemania o en Inglaterra a las cinco de la tarde casi todo el mundo está en casa. De hecho, para las seis hay bastantes periódicos que han cerrado la edición del día siguiente. Aquí en España eso no ocurre. El día se estira y hay ruedas de prensa hasta las ocho o las nueve. Por ejemplo, en algunas sesiones parlamentarias, los políticos debaten hasta las doce de la noche. Y los periodistas tenemos que cubrir ese evento. Forma parte de nuestra cultura y eso es muy difícil de cambiar".

Sin embargo, y como ocurría con los sueldos, los prolongados horarios tampoco influyen decisivamente en la insatisfacción de los periodistas españoles. En líneas generales, desearían tener más tiempo para compartirlo con familiares y 
amigos. Pero, a la vez, se dejan absorber diariamente por las noticias y por el frenético devenir de la actualidad. Como dice Juan Luis Cebrián, "el periodista vocacional, el que vive la profesión, no mira nunca el reloj cuando está haciendo un reportaje. Hasta que no termina de encontrar todos los datos, hasta que no redacta la última línea, no se marcha a casa". Y la satisfacción de haber culminado una buena crónica resarce las largas horas de trabajo.

\section{3.- Satisfacción y organización del trabajo}

De lo visto hasta ahora se deduce que la escasez de sueldos y las estiradas jornadas laborales, aun siendo importantes, no son decisivas a la hora de explicar la insatisfacción laboral de los periodistas, algo que han constatado también otros autores como Barrett (1984) y Weaver y Wilhoit (1996) ${ }^{43}$. Tampoco lo son, según nuestro sondeo, otros factores como la edad, el número de años en la profesión, el cargo desempeñado... Ninguno de estos elementos contribuye a delimitar claramente las razones de satisfacción o de insatisfacción.

Por tanto, ¿a qué se debe que un periodista esté más contento o satisfecho con su trabajo? Los resultados de correlaciones estadísticas entre las distintas variables del sondeo permiten llegar a una conclusión contundente: la satisfacción laboral está íntimamente relacionada con la descentralización del poder en la redacción. El dato que mejor explica la satisfacción profesional es el tipo de redacción en la que se trabaja ${ }^{44}$; por lo tanto, hablamos de un factor enraizado en las propias condiciones profesionales. Coincide que los más satisfechos son los que desempeñan su función en una redacción más participativa; y los menos, aquellos periodistas que trabajan en redacciones jerárquicas ${ }^{45}$.

Esta conclusión está en la línea de estudios realizados hasta el momento en otros países con mayor tradición en investigación sociológica de la profesión periodística. En 1966, Herzberg comprobó, tras más de diez años de análisis de las

43 Cfr. BARRETT, Grace (1984): “Job satisfaction among newspaper women”, Journalism Quarterly, n. 61, pp. 593-599; WEAVER, D. y WILHOIT, C. (1996): The American Journalist, New Jersey: Lawrence Erlbaum.

44 Las correlaciones estadísticas fueron las siguientes:

\begin{tabular}{|l|c|l|l|l|l|c|}
\hline & Ingresos & $\begin{array}{l}\text { Horas de } \\
\text { trabajo }\end{array}$ & Edad & $\begin{array}{l}\text { Número de medios en } \\
\text { los que ha trabajado }\end{array}$ & $\begin{array}{l}\text { Tiempo en puestos } \\
\text { de responsabilidad }\end{array}$ & $\begin{array}{l}\text { Descentralización de } \\
\text { la redacción }\end{array}$ \\
\hline Satisfacción & 0,054 & $-0,025$ & 0,079 & $-0,043$ & 0,127 & $0,168 * *$ \\
\hline
\end{tabular}

La única correlación estadísticamente significativa es la compuesta por las variables "satisfacción” y "descentralización de la redacción".

45 Una de las cuestiones que se incluyeron en el sondeo era: “Cómo calificaría la organización de su redacción?”. A continuación se proponía una escala de 1 a 6 , donde 1 era jerárquica y 6 participativa. Se entendía por jerárquica aquella redacción estrictamente compartimentada, donde los directores y jefes de sección marcan todas las pautas a seguir y en las que los redactores tienen escasa capacidad de iniciativa. Por el contrario, la redacción participativa es aquella que huye de esta rigidez y en la que los temas se discuten y proponen de forma compartida. Las contestaciones a esta pregunta fueron muy ajustadas: un 54,7 por ciento de la muestra afirmó que su redacción está jerarquizada y un 45,3 por ciento que es participativa. 
actitudes profesionales, que factores como el reconocimiento del trabajo o el hecho de desarrollar su labor en un entorno dinámico con posibilidades de participación eran factores que influían decisivamente en la satisfacción de cualquier profesional ${ }^{46}$. En 1978, Shaver evidenció igualmente que la posibilidad de participar en la redacción y el hecho de sentir reconocido el propio trabajo (incluso con la opción de ascender profesionalmente) eran factores decisivos en ese índice de satisfacción de los periodistas ${ }^{47}$. En 1984, en una encuesta realizada a mujeres periodistas en Estados Unidos, se constató que los factores intrínsecos (organización de la redacción, valoración del trabajo, autonomía...) eran más importantes que los extrínsecos (sueldos, horarios...) a la hora de valorar la satisfacción profesional ${ }^{48}$. En 1993, Stamm y Underwood constataron la percepción de que los periodistas se sienten más satisfechos en medios de pequeñas dimensiones, en los que existen mayores cauces de participación y de diálogo entre los miembros de la redacción y en los que hay menos burocracia y restricciones a la autonomía periodística ${ }^{49}$, una conclusión muy similar a la obtenida años antes, en 1976, en un sondeo a 1.300 periodistas llevado a cabo por Johnstone, Slawski y Bowman ${ }^{50}$. Demers demostró en 1994 que el índice de satisfacción profesional era más elevado en las redacciones descentralizadas, caracterizadas por un mayor grado de autonomía para los periodistas ${ }^{51}$. Un año más tarde, Pollard también concluyó que la satisfacción depende tanto del tipo de redacción como del diálogo frecuente con los supervisores y la percepción de autonomía del periodista ${ }^{52}$. En 1996, Weaver y Wilhoit constataron que los mejores predictores para evaluar la satisfacción profesional de los informadores eran la existencia de comunicación entre los miembros de la redacción, especialmente entre los redactores y los jefes, la percepción de autonomía y la flexibilidad en la organización del trabajo ${ }^{53}$.

46 Cfr. HERZBERG, Frederick (1966): Work and the Nature of Man, Nueva York: Thomas Y. Crowell Publishers, pp. 71-167.

47 Cfr. SHAVER, Harold C. (1978): "Job Satisfaction and Dissatisfaction Among Journalism Graduates", Journalism Quarterly, n. 55, pp. 54-61.

48 Cfr. BARRETT, Grace (1984): “Job satisfaction among newspaper women”, Journalism Quarterly, n. 61, pp. 593-599.

49 Cfr. STAMM, Keith y UNDERWOOD, Doug (1993): “The Relationship of Job Satisfactioin to Newsroom Policy Changes", Journalism Quarterly, n. 70, otoño, pp. 528-541.

50 Cfr. JOHNSTONE, John W.C., SLAWSKI, Edward y BOWMAN, William (1976): The News People: A Sociological Portrait of American Journalists and Their Work, Urbana: University of Illinois Press, pp. 133-156.

51 Cfr. DEMERS, David P. (1994): "Effect of organizational size on job satisfaction of top editors at U.S. dailies", Journalism Quarterly, vol. 71, n. 4, pp. 914-925.

52 Cfr. POLLARD, George (1995): "Job satisfaction among newsworkers: the influence of professionalism, perceptions of organizational structure, and social attitudes", Journalism and Mass Communication Quarterly, vol. 72, n. 3, pp. 682-697.

53 Cfr. WEAVER, D. y WILHOIT, C. (1996): The American Journalist, New Jersey: Lawrence Erlbaum; WEAVER, D. (ed.) (1998): The Global Journalist, New Jersey: Hampton Press, pp. 88-92. 
En definitiva, de todos estos estudios se puede extraer la conclusión de que la autonomía profesional, la participación, el diálogo en las redacciones y la valoración del trabajo son factores que influyen decisivamente en la satisfacción de los periodistas. Y como hemos apuntado, en nuestro sondeo la relación entre satisfacción y organización de la redacción queda patente en numerosas variables. Todas las preguntas formuladas referidas a la organización del trabajo se relacionan claramente con el índice de satisfacción profesional. Las cotas más bajas se encuentran entre aquellos periodistas que dicen "tener un trabajo que consiste sólo en reproducir lo que unos y otros dicen que pasa, más que en averiguar lo que pasa"; "que hay poca generación de temas propios"; "que se va a remolque de lo que otros medios más poderosos consideran importante"; y "que afirman pensar más en cómo cubrir la sección que en levantar noticias".

Por el contrario, hay satisfacción cuando la organización de la redacción permite levantar informaciones, no ir a remolque de los demás, generar temas propios, ejercitar la creatividad.

Indudablemente, si tener a la redacción satisfecha es una de las mejores garantías de éxito para cualquier medio de comunicación, los directivos y responsables de estos medios deberían tener en cuenta esta conclusión. Una organización demasiado vertical adocena, acomoda y duerme a la redacción. La participación, por el contrario, despierta a los redactores, los hace combativos, activos, creativos y buscadores de información. Cuando al periodista se le permite desarrollar su trabajo en un ambiente de iniciativa alejado de la rutina, está satisfecho. Y está dispuesto a aguantar el estrés, las prisas, la tensión y hasta la escasez económica.

En conclusión, los resultados aportados en el presente artículo vienen a constatar la importancia de la organización de las redacciones en el índice de satisfacción profesional de los periodistas, por encima incluso de otros factores más relacionados con sus condiciones laborales o salariales. Esta constatación está en la línea de las aportaciones teóricas ofrecidas en otros países de nuestro entorno, y contribuye a definir con mayor precisión la naturaleza y las especificidades de la profesión periodística. 